\title{
A Simple Technique to Readout and Characterize Coupled MEMS Resonators
}

\author{
Guowei Tao and Bhaskar Choubey, Senior Member, IEEE
}

\begin{abstract}
Highly sensitive microelectromechanical system (MEMS) resonators have been used for extensive applications like mass sensing. However, these arrays are prone to process variations and are difficult to characterize due to lack of connections with each resonator. Coupled array of such sensors can enhance functionality while reducing interconnections. This scheme allows a number of sensors to be read out using a single input/output channel. By perturbing the spring constant of one resonator, two sets of eigenvalues can be measured to reconstruct the system matrix of the coupled system. However, perturbation on MEMS devices can introduce non-linear or physical change. To prevent such undesired change, this paper proposes a technique to couple an electrical resonator to an array of MEMS resonators. Two sets of eigenvalues can therefore be measured by simply connecting/disconnecting the electrical resonator. Errors introduced by frequency noise, array size, coupling strength and damping have been analysed. The successful coupling between electrical and mechanical resonators brings huge promise for integrated MEMS devices that could be used for single input/output multisensor and monitoring variations in MEMS fabrication.
\end{abstract}

Index Terms-MEMS, coupled sensors, micro/nano resonators, inverse eigenvalue analysis.

\section{INTRODUCTION}

$\mathbf{M}$ ICRO/NANO resonators have been used for numerous applications including mass sensor [1], [2], accelerometer [3], [4], gyroscope [5], [6], RF filter [7], [8], process characterisation [9], [10] and material characterisation [11], [12]. MEMS based mass sensors, for instance, have been used to detect chemical and biomedical substances by providing a frequency shift proportional to the mass change of the accreted molecules [13], [14]. With very small resonators being built due to advances in nanofabrication, they can provide ultra-high sensitivity [15]-[17]. These advances have also enabled the potential of making several sensors on a single substrate and thereby providing multifunction sensors [18]-[21]. However, it is difficult to use these due to separate drive and readout connections required for each resonator, which increase the number and total size of bond pads. The bond pads do not shrink at the same rate as the nanofabricated devices and hence they are consuming increasing percentage of chip area [22]. Therefore it is very important to reduce their number to save costly fabrication area. Multi input/output devices also increase the bandwidth.

One solution to both problems is to use an array of coupled resonators [23]-[27]. Resonators can be coupled parasitically [12], mechanically [7] or electrostatically [23]. Such an array

The authors are with the Department of Engineering Science, University of Oxford, Oxford OX13PJ, UK (e-mail: guowei.tao@eng.ox.ac.uk; bhaskar.choubey@eng.ox.ac.uk).

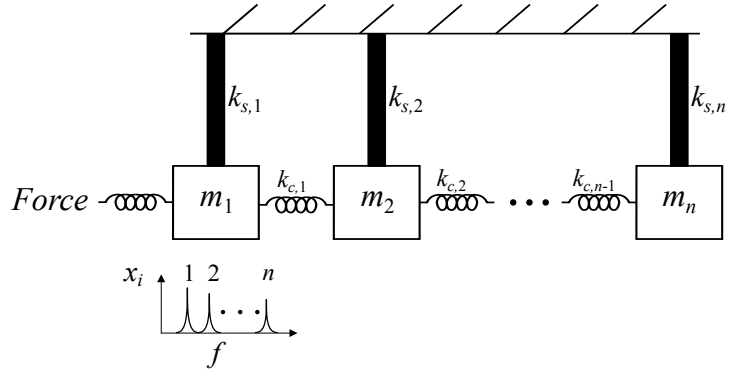

Fig. 1. An array of $n$ nearest-neighbour coupled resonators with a typical frequency response showing $n$ peaks.

of $n$ coupled resonators can be modelled by the second order differential equation:

$$
\ddot{\mathbf{x}}+\mathbf{B}_{\mathbf{n}} \dot{\mathbf{x}}+\mathbf{S}_{\mathbf{n}} \mathbf{x}=\mathbf{F}
$$

where $\mathbf{x}$ is a column vector of the displacements of all resonators, $\mathbf{F}$ is the applied force and $\mathbf{S}_{\mathbf{n}}$ denotes the system matrix of the array. $\mathbf{B}_{\mathbf{n}}$ is a normalized damping matrix, which can be ignored when quality factor $(Q)$ is high enough. The eigenvalues of this system matrix represent the resonance frequencies of the system. These can be measured from the response of any one resonator due to collective behaviour of the coupled system. When the mass or spring constant of any of these resonators changes, all eigenvalues change in a unique way [28]. Monitoring the changes from the response of any resonator is often enough to identify the changed resonator as well as the amount of change [24]. Driving/reading from only one resonator is therefore sufficient to monitor changes in a large coupled array of resonators. Hence, the system becomes a single input single output multiplexed array of sensors [29]. Apart from mass sensing, coupled micro/nano resonators have been investigated by many researchers for applications such as accelerometer [4], gyroscope [6], process characterisation [10], filter design [7] and Young's Modulus determination [12].

Fig. 1 shows an array of $n$ nearest-neighbour coupled resonators. The system matrix $\mathbf{S}_{\mathbf{n}}$ of this array is a symmetric tridiagonal (Jacobian) matrix:

$$
\mathbf{S}_{\mathbf{n}}=\left[\begin{array}{cccc}
\frac{k_{s, 1}+k_{c, 1}}{m_{1}} & \frac{-k_{c, 1}}{\sqrt{m_{1} m_{2}}} & \ldots & 0 \\
\frac{-k_{c, 1}}{\sqrt{m_{1} m_{2}}} & \frac{k_{s, 2}+k_{c, 1}+k_{c, 2}}{m_{2}} & \ldots & 0 \\
\vdots & \vdots & \ddots & \vdots \\
0 & 0 & \frac{-k_{c, n-1}}{\sqrt{m_{n-1} m_{n}}} \frac{k_{s, n}+k_{c, n-1}}{m_{n}}
\end{array}\right]
$$


where, $k_{s, i}$ and $m_{i}$ are the spring constant and mass of the $i^{\text {th }}$ resonator, respectively. $k_{c, i}$ is the coupling strength between the $i^{\text {th }}$ and the $(i+1)^{\text {th }}$ resonator. The elements of this matrix, $S_{n}(i, j)$ for $i, j=1, \cdots, n$ are given by:

$$
S_{n}(i, j)=\left\{\begin{array}{cl}
\frac{k_{s, i}+k_{c, i}}{m_{i}} & \forall i=j=1 \\
\frac{k_{s, i}+k_{c, i-1}}{m_{i}} & \forall i=j=n \\
\frac{k_{s, i}+k_{c, i-1}+k_{c, i}}{m_{i}} & \forall i=j \neq 1 \mid n \\
\frac{-k_{c, i}}{\sqrt{m_{i} m_{j}}} & \forall i=j-1 \\
\frac{-k_{c, i-1}}{\sqrt{m_{i} m_{j}}} & \forall i=j+1 \\
0 & \forall \text { others }
\end{array}\right.
$$

However, these parameters are dependent on manufacturing conditions often poorly modelled by simulators. Furthermore, microfabrication is known to introduce variability in manufactured devices and hence the parameters of different resonators are often different, even when they are made on the same substrate and with the identical design [30], [31]. Therefore, there is a need to extract the system matrices from experimental responses before using these coupled arrays as sensors. Previously proposed techniques to characterize coupled system use either the inherent non-linearity of the system or physically change the system [10], [12]. However, these are often impractical and impossible for a large number of resonators array.

The objective of this paper is to propose a simple yet effective technique to readout and characterize coupled sensors, which can be used for all kinds of resonator based MEMS applications. The approach avoids unwanted physical perturbation by coupling an additional electrical resonator to the MEMS array. We also present a different inverse eigenvalue analysis method to minimize the effect of measurement error.

The paper is organised as follows: section II provides theoretical analysis of the inverse eigenvalue technique. A new algorithm to extract the system matrix of the electricalmechanical coupled array is introduced. To verify this novel technique, a series of experiments were performed to characterize two coupled MEMS resonators in section III. Section IV studies the effect of noise arisen from the output frequency measurement using Monte Carlo procedure. It also discusses the effect of damping in practical applications. Section V provides the conclusion.

\section{INVERSE EIGENVALUE ANALYSIS}

For an array of $n$ nearest-neighbour coupled MEMS resonators, there are $2 n-1$ terms in the system matrix, which are impossible to extract from the $n$ measured resonance frequencies of the system. Therefore we need a minimum of $2 n-1$ measurements to extract their values. One set of $n$ eigenvalues $\mu_{i}$ of the matrix is readily available from the resonance frequencies $f_{i}$ as

$$
\mu_{i}=\left(2 \pi f_{i}\right)^{2}
$$

Another set of eigenvalues is hence desired for inverse eigenvalue analysis. To obtain another set of eigenvalues, a small perturbation can be introduced to the system. In previous approaches, perturbation was performed by removing a section of one resonator using focused ion beam (FIB) milling [12], or by applying additional DC voltage to introduce nonlinear spring softening in electrostatically driven resonators [28]. The former introduces a permanent damage to the device, while the later introduces a non-linear change and is only possible with electrostatically actuated devices.

In this paper, we present an alternative technique to prevent physical or non-linear change by coupling an additional resonator. One can then use a modified inverse eigenvalue analysis technique. In practice, physically adding a mechanical resonator is impossible once the devices have been fabricated. Nevertheless, an external electrical resonator with similar resonance frequency can be coupled to the array of MEMS resonators. Two sets of eigenvalues can then be recorded by simply connecting and disconnecting the electrical resonator, without influencing the mechanical array. Coupling an extra resonator makes inverse eigenvalue analysis applicable to any array of MEMS resonators, irrespective of the drive or excitation mechanism.

Coupling a new resonator with similar resonance frequency to an array of $n$ MEMS resonators forms a new array with $n+1$ members and therefore produces another set of eigenvalues, which can be used to find all elements of the system matrix with the previous $n$ measurements. Coupling an additional resonator to the last member of the array creates a new $n+1$ array with system matrix $\mathbf{S}_{\mathbf{n}+\mathbf{1}}$. Such a new array has $n+1$ eigenvalues $\lambda_{i}$, which can be recorded from the response of any one of the MEMS resonators or even simply the external electrical resonator.

$\mathbf{S}_{\mathbf{n}}$ is the left principal submatrix of the Jacobian matrix $\mathbf{S}_{\mathbf{n}+\mathbf{1}}$. Hence, de Boor and Golub's algorithm based on orthogonal polynomials [32] can be applied without extracting eigenvectors. The algorithm starts by first computing a set of weights $w_{i}$ from the measured eigenvalues $\lambda$ and $\mu$ :

$$
w_{i}=\prod_{j=1}^{n} \frac{\lambda_{i}-\mu_{j}}{\lambda_{i}-\lambda_{j+1}} \quad i=1, \cdots, n+1
$$

A set of orthogonal polynomials, related to a reflected version of $\mathbf{S}_{\mathbf{n}+\mathbf{1}}$ are then evaluated, with the first two being

$$
\begin{aligned}
& p_{0}\left(\lambda_{i}\right)=1 \quad i=1, \cdots, n+1 \\
& p_{1}\left(\lambda_{i}\right)=\lambda_{i}-\frac{\sum_{j=1}^{n+1} w_{j} \lambda_{j}}{\sum_{j=1}^{n+1} w_{j}} \quad i=1, \cdots, n+1
\end{aligned}
$$

Since the values of $p_{0}\left(\lambda_{i}\right)$ and $p_{1}\left(\lambda_{i}\right)$ are known, threeterm recurrence relationships can be written to compute the elements of the system matrix as well as the subsequent orthogonal polynomials $p_{k+1}\left(\lambda_{i}\right)$ iteratively. If the diagonal elements $S_{n+1}(i, i)$ are $d_{i}$, and off-diagonal elements $S_{n+1}(i, i+1)=S_{n+1}(i+1, i)$ are $o_{i}$, then

$$
\begin{aligned}
& d_{n+2-k}=\frac{\sum_{i=1}^{n+1} \lambda_{i} w_{i} p_{k-1}^{2}\left(\lambda_{i}\right)}{\sum_{i=1}^{n+1} w_{i} p_{k-1}^{2}\left(\lambda_{i}\right)} \quad k=1, \cdots, n+1 \\
& o_{n+1-k}^{2}=\frac{\sum_{i=1}^{n+1} w_{i} p_{k}^{2}\left(\lambda_{i}\right)}{\sum_{i=1}^{n+1} w_{i} p_{k-1}^{2}\left(\lambda_{i}\right)} \quad k=1, \cdots, n
\end{aligned}
$$




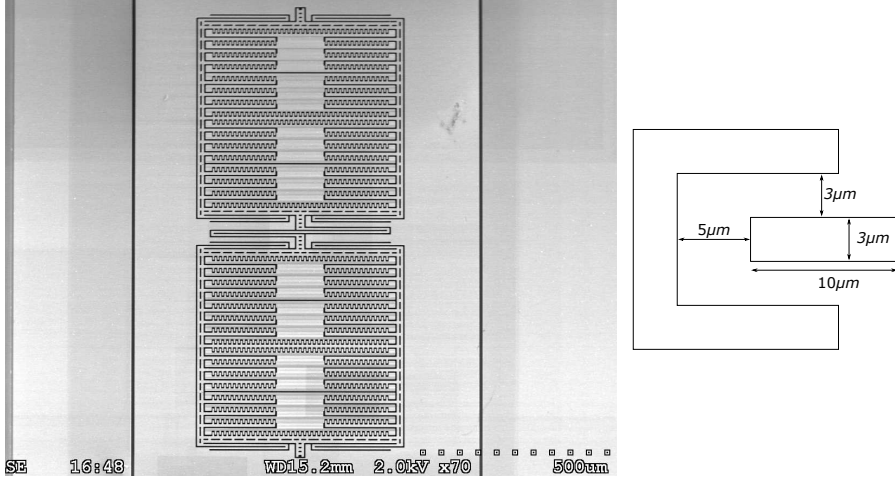

Fig. 2. (a) SEM image of two coupled MEMS resonators made from electrostatically driven comb capacitors. (b) The dimensions of each finger of the comb.

The orthogonal polynomials can also be iteratively computed for $k=2, \cdots, n-1$ by using

$$
p_{k}\left(\lambda_{i}\right)=\left(\lambda_{i}-d_{n+2-k}\right) p_{k}\left(\lambda_{i}\right)-o_{n+2-k}^{2} p_{k-2}\left(\lambda_{i}\right)
$$

It is worth noting that the algorithm starts filling the matrix from $S_{n+1}(n+1, n+1)$ towards $S_{n+1}(1,1)$. Hence both diagonal and off-diagonal elements of $\mathbf{S}_{\mathbf{n}+\mathbf{1}}$ are derived. Since $\mathbf{S}_{\mathbf{n}}$ is the left principal submatrix of $\mathbf{S}_{\mathbf{n}+\mathbf{1}}$, the $n$ MEMS resonators array can be fully characterized.

\section{EXPERIMENT AND RESULTS}

Mathematically, this technique can be applied to a large number of resonators as long as the eigenfrequencies can be measured. However, for a rapid verification, we have used the resonators shown in Fig. 2, where neither the coupling nor the resonators are optimally designed.

\section{A. MEMS Resonators}

To experimentally verify the technique, an array of two MEMS resonators was tested. Fig. 2 shows the SEM photograph and dimensions of the two resonators. These resonators are opposing combs arranged in a push-pull configuration, excited by differential drive/readout circuits. Neighbouring resonators are coupled together through a weak mechanical spring. Experiments were taken under low pressure to enable high quality factor.

Such a system has been previously characterized using inverse eigenvalue analysis with Lanczos' algorithm [10]. This however requires introduction of non-linear change in the system. A change in the spring constant of the MEMS resonator was provided by using the electrostatic driving force $F_{E S}$. This force is position dependent and will change the effective spring constant. For a parallel plate with a static capacitance $C_{0}$ and initial displacement $x_{0}$, if it is displaced by $x$, then the electrostatically induced stiffness is determined by:

$$
K_{E}=\frac{d F_{E S}}{d x}=-V^{2} \frac{C_{0}}{x_{0}^{2}}\left(1+\frac{3 x}{x_{0}}+\cdots\right)
$$

The electrostatically induced stiffness $K_{E}$ is linearly dependent on the square of applied DC voltage. By changing the

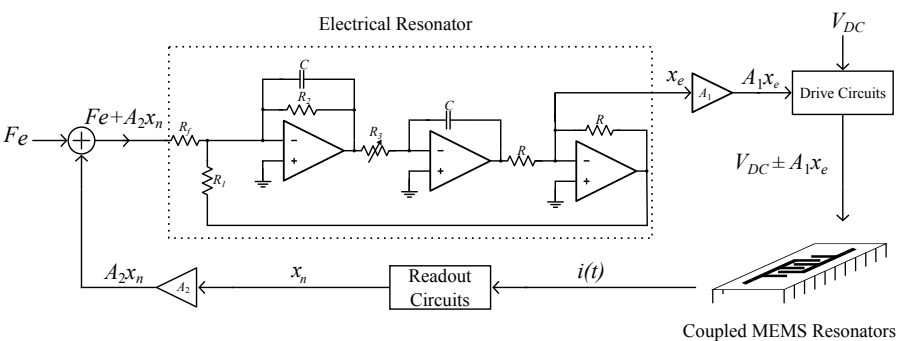

Fig. 3. Full experiment setup for coupling electrical resonator with MEMS resonators array.

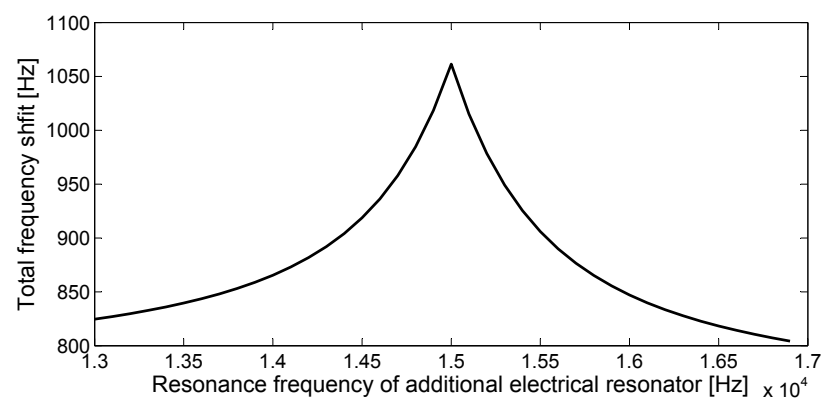

Fig. 4. Total frequency shift after coupling an additional electrical resonator with different resonance frequencies to an array of two MEMS resonators.

applied voltage, two sets of eigenvalues can be derived and used for inverse eigenvalue analysis, without requiring the knowledge of the exact value of the spring constant change. The resonators used in this paper are very similar to those used in [10] and hence, can be characterized using a change in spring constant as well. This provides a benchmark to compare the new technique with the previously reported work. Hence, the nonlinearity of equation 9 is only used to verify the technique.

\section{B. Electrical Resonator}

The proposed electrical resonator to be coupled to the MEMS resonators can be a very simple electrical resonator [33], [34], which is shown inside the dotted box in Fig. 3. The centre frequency and quality factor of this electrical resonator can be easily adjusted. The circuit is modelled by

$$
\ddot{x}_{e}+\frac{1}{R_{2} C} \dot{x}_{e}+\frac{1}{R_{1} R_{3} C^{2}} x_{e}=\frac{1}{R_{3} R_{f} C^{2}} F_{e}
$$

where $F_{e}$ is the driving voltage and $x_{e}$ is the output voltage. The centre frequency

$$
f_{0}=\left(2 \pi \sqrt{R_{1} R_{3}} C\right)^{-1}
$$

can be adjusted by changing the resistance values. The circuit is designed to provide low-damping $(Q>200)$ by selecting suitable passive elements.

This circuit was coupled to the array of MEMS resonators by converting its output to electrostatic force to drive one of the MEMS resonators. Conversely, the mechanical output of the same MEMS resonator was converted to an electrical signal to feed back to the circuit. Once coupled, readouts from both the electrical circuit and the mechanical resonators 


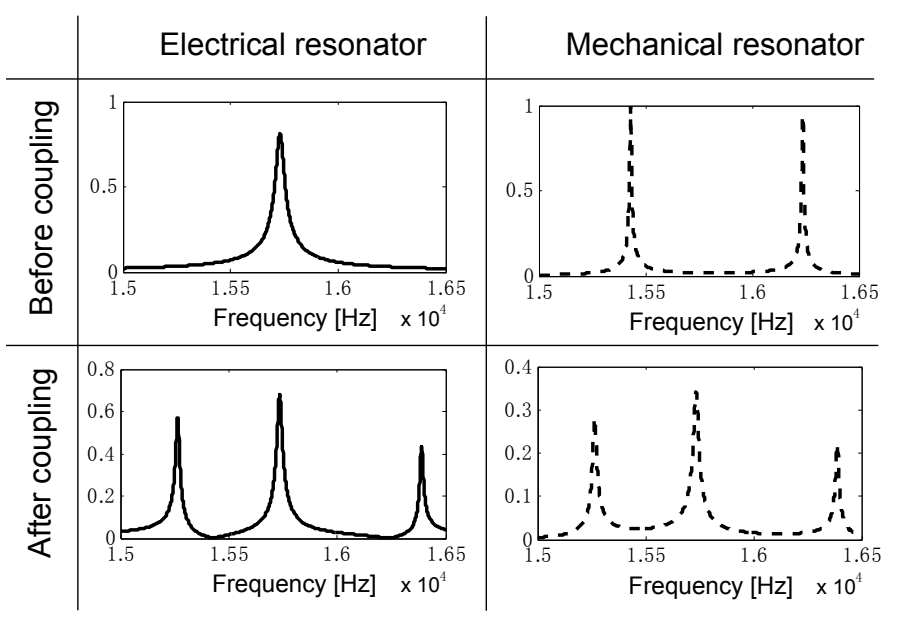

Fig. 5. Simulated responses from electrical and mechanical resonators before/after coupling.

contain $n+1$ resonant peaks. These can be measured from the response of the mechanical resonator or better still, the electrical resonator.

Fig. 3 describes the experimental setup for coupling and recording from the electrical and mechanical resonators. The electrical resonator output $x_{e}$ was amplified and superimposed on a DC voltage to generate a pair of differential signals. The differential signals were subsequently applied to the opposing combs of one MEMS resonator to electrostatically drive it. The readout circuits for this resonator measured the change in capacitance producing a current output, which was then converted into a voltage by a transimpedance amplifier. The output was then amplified and added to the driving voltage of the electrical circuit. The gains in the two signal paths, electrical to MEMS and MEMS to electrical, were carefully adjusted to be similar.

To find the required resonance frequency of the electrical resonator, a simulation was undertaken by coupling a resonator with varying resonance frequencies to an array of two identical resonators with resonance frequency of $15 \mathrm{KHz}$. The ratio of the coupling spring to the principal spring, i.e. the relative coupling strength, was $5 \%$ depicting weak coupling condition. It was observed from the simulations shown in Fig. 4, that more measurable total frequency shift for all resonators is achieved by setting the resonance frequency of the additional circuit around $15 \mathrm{KHz}$. This indicates that the centre frequency of the electrical resonator should approximate that of the mechanical resonators to enable more obvious shift phenomenon and fewer readout errors.

\section{Frequency Response and System Matrix}

Fig. 5 shows four simulated responses with typical values recorded previously [28]. These include the responses of one of the two coupled MEMS resonators, an electrical resonator before and after coupling, respectively.

Experimentally, with the electrical resonator coupling to the mechanical resonators, a drive voltage $F_{e}$ was applied to the electrical resonator with a frequency sweep from 15 to 16.5 $\mathrm{KHz}$ and a resolution of $1 \mathrm{~Hz}$. The measured response of

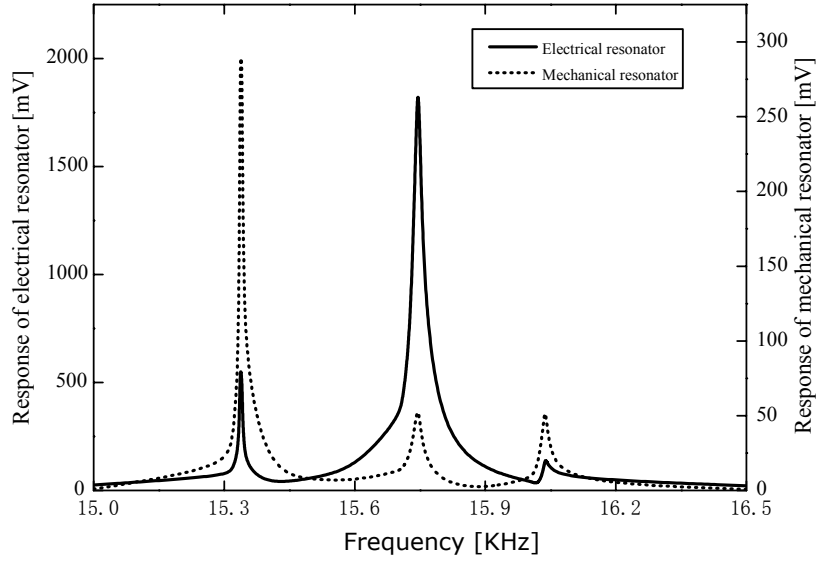

Fig. 6. Experimentally measured responses from electrical and mechanical resonators after coupling.

both the electrical resonator as well as one of the mechanical resonators, as presented in Fig. 6, showed three collective resonance frequencies at $(15343,15745,16037) \mathrm{Hz}$. These were used to find the three eigenvalues of $\mathbf{S}_{3}$. The electrical resonator was then disconnected from the two mechanical resonators and drive voltage was directly applied to one of the two coupled MEMS resonators. From the mechanical readout, two resonant peaks at $(15371,16025) \mathrm{Hz}$ were observed. Using the new technique discussed in the previous section, the system matrix $\mathbf{S}_{\mathbf{3}}$ was extracted to be:

$$
\mathbf{S}_{3}=10^{9} \times\left[\begin{array}{ccc}
9.9280 & -0.3552 & 0 \\
-0.3552 & 9.5375 & -0.1465 \\
0 & -0.1465 & 9.7681
\end{array}\right]
$$

It is the diagonal elements of the matrix that contain the information about individual resonators and hence are more important. The diagonal elements of $\mathbf{S}_{\mathbf{3}}$ suggest that centre frequency of the electrical resonator is $15730 \mathrm{~Hz}$. A comparison to the actual resonance frequency of the electrical resonator $(15723 \mathrm{~Hz})$ shows a small relative error of $0.04 \%$.

\section{Validation}

Two different approaches were used to validate the results. In the first approach, perturbation analysis was performed in the conventional way using DC voltage based nonlinearity in spring, as described in equation 9. The electrical resonator was disconnected. The MEMS system was perturbed by changing the applied DC voltage on one of the resonators. The two sets of resonance frequencies before and after perturbation were measured to be $(15371,16024) \mathrm{Hz}$ and $(15388,16031) \mathrm{Hz}$, respectively. These two sets of eigenvalues were used to extract the system matrix

$$
\mathbf{S}_{\mathbf{2}}=10^{9} \times\left[\begin{array}{cc}
9.9000 & -0.3682 \\
-0.3682 & 9.5643
\end{array}\right]
$$

As $\mathbf{S}_{\mathbf{2}}$ is the left principal submatrix of $\mathbf{S}_{\mathbf{3}}$, the new technique produces comparable results. As mentioned before, the nonlinear spring constant change introduced by the conventional method could affect the accuracy of measurement. 


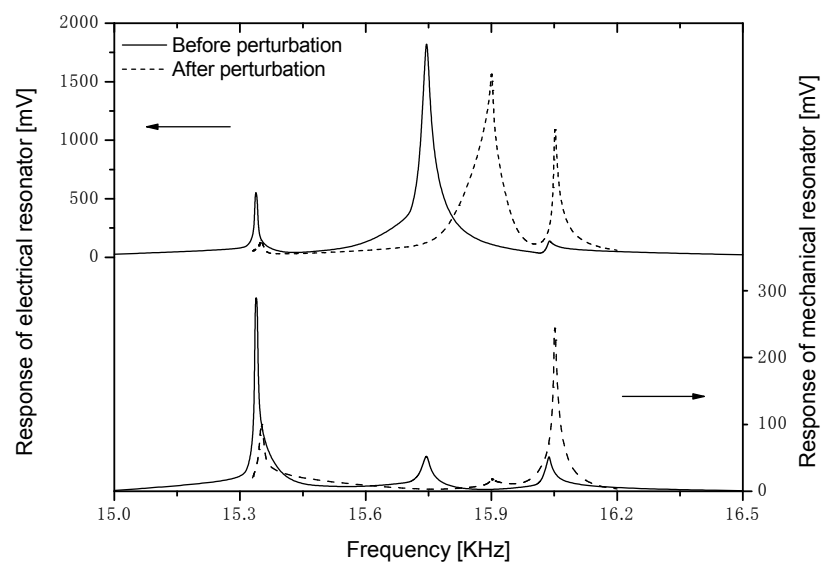

Fig. 7. Measured responses of electrical resonator and mechanical resonator before and after perturbation upon the centre frequency of the electrical resonator.

Hence to further validate the result, a second approach using perturbation analysis on the complete electrical-mechanical array was also performed. When the electrical resonator was coupled to the MEMS array, perturbation was induced by simply changing the centre frequency of the electrical resonator. A frequency shift for all three peaks was observed in Fig. 7 to give another three eigenvalues. With these two sets of eigenvalues before and after perturbation, the system matrix $\mathbf{S}_{\mathbf{3}}$ was once again calculated:

$$
\mathbf{S}_{\mathbf{3}}=10^{9} \times\left[\begin{array}{ccc}
9.9195 & -0.3634 & 0 \\
-0.3634 & 9.5417 & -0.1334 \\
0 & -0.1334 & 9.7726
\end{array}\right]
$$

The diagonal elements of the above system matrix show excellent agreement with the system matrix in equation 12 , indicating the feasibility and accuracy of the proposed new technique.

\section{ERROR ANALYSIS}

\section{A. Noise Effect}

Experimental results show that the derived natural resonance frequencies vary from the designed values, which are caused by the process variations widely seen in microelectronics industry. However, the effectiveness of the proposed inverse eigenvalue analysis is dependent on the accuracy of the measured data. Better data give better eigenvalues that can be used to accurately reconstruct the system matrix. Measurement errors, which are caused by random temporal noise or environment factors, have a significant impact on the accuracy of inverse eigenvalue analysis. Hence, it is important to analyse the effect of noise with numerical experiment.

Monte Carlo procedure has been used to study Gaussian random noise. Electronic circuit noise is an example of Gaussian random noise. For coupled resonators system, the effect of noise and error can be modelled by superimposing a Gaussian distributed frequency noise on the actual resonance frequencies of the system. Such Gaussian noise has a mean value of zero and a particular standard deviation depending on the quality of readout channel. Numerical experiments

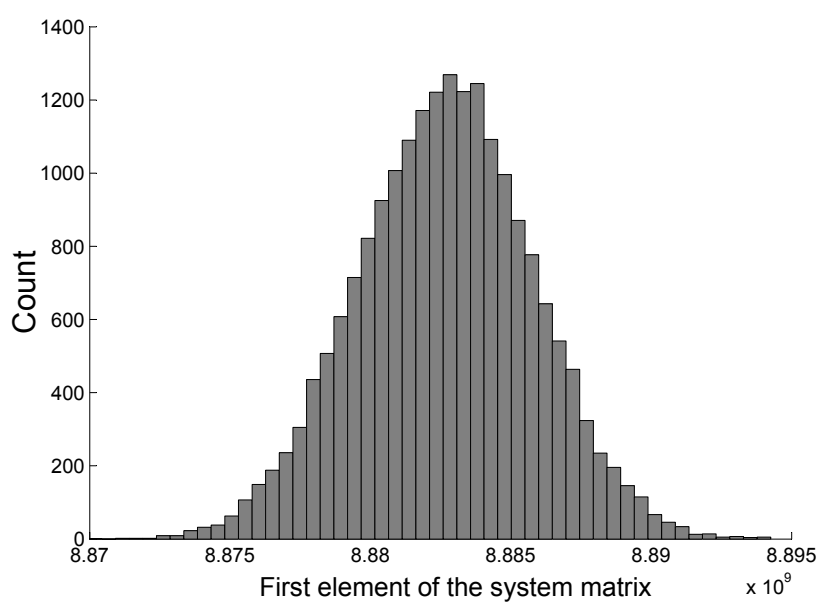

Fig. 8. Monte Carlo analysis of the spread in the extracted $S_{3}(1,1)$ when an $1 \mathrm{~Hz}$ standard deviation Gaussian noise was added to measurement.

were conducted by several Monte Carlo runs to simulate the influence of measurement error on the finally derived system matrix. In each Monte Carlo run, two sets of eigenvalues corrupted by Gaussian random noise were simulated. These two sets of eigenvalues could then be used to reconstruct the system matrix of the array using inverse eigenvalue analysis. A numerical experiment with $k^{\text {th }}$ run produces $k$ varied system matrices.

A chain of three nearest-neighbour coupled resonators were simulated, each with a natural resonance frequency of 15 $\mathrm{KHz}$ and a relative coupling strength of $5 \%$. Another $15 \mathrm{KHz}$ resonator was coupled to these three resonators. Fig. 8 shows the first element of extracted system matrix using Monte Carlo procedure considering the existence of the measurement noise. The simulated noise is Gaussian distributed with a mean value of zero and a standard deviation of $1 \mathrm{~Hz}$. These lead to a Gaussian error in extracted system matrix elements. For further investigation, a metric is needed to characterize the effect of noise. The percentage spread of one matrix element $S_{n}(i, j)$ could be represented by the ratio of its standard deviation and mean for the $k$ runs:

$$
E_{r}(i, j)=\frac{\operatorname{std}\left[S_{n, 1}(i, j), \cdots, S_{n, k}(i, j)\right]}{\operatorname{mean}\left[S_{n, 1}(i, j), \cdots, S_{n, k}(i, j)\right]} \times 100 \%
$$

As only the diagonal elements of the system matrix are related to the characteristic of each resonator, a simple metric defined by the root mean square deviation of the diagonal elements can be used as an error metric:

$$
D_{r}=\frac{\sqrt{\sum_{i=1}^{n} E_{r}^{2}(i, i)}}{n}
$$

With increase in the number of elements, noise of each element accumulates. In addition, the measurement noise, which is caused by the sum of readout circuit, temperature variation as well as numerical error, also limits the performance of inverse eigenvalue analysis. The accuracy of the proposed technique has been studied considering these two factors. The percentage errors represented by the root mean square deviation of the percentage spread of the diagonal elements were analysed, as shown in Fig. 9, where we compare the noise 


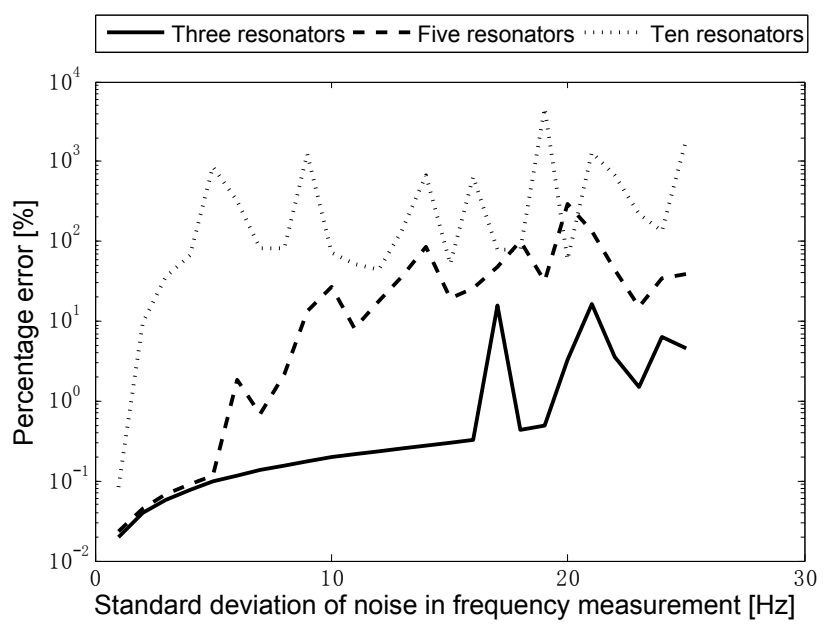

Fig. 9. Percentage error of system matrix with different noise levels for three, five and ten resonators (before correction).

performance of systems with three, five and ten resonators. The percentage error increases with the number of resonators and the standard deviation of noise. It is worth noting that, for a large number of resonators and high noise level, the technique becomes extremely unstable with sharp increase and wild fluctuation. For example, the technique works well under $15 \mathrm{~Hz}$ noise level for a three-resonator system, with percentage error predictable and well-controlled. However, as the noise further increases, the error fluctuates wildly and increases above $1 \%$. For five resonators, the error becomes very high just after $5 \mathrm{~Hz}$ noise. For ten resonators, percentage error increases dramatically for even small amount of noise. In large array applications like mass sensors, such error can easily deteriorate the accuracy of sensing and hence needs to be avoided. However, these errors can be reduced by a very simple correction mechanism.

For the proposed inverse eigenvalue analysis aforementioned in Section II, it is important to ensure that the shifts in the individual eigenfrequencies are larger than the frequency noise during measurement. Shifts in eigenfrequencies should not break the interweaving property of the eigenvalues:

$$
\lambda_{1}<\mu_{1}<\lambda_{2}<\mu_{2}<\cdots<\lambda_{n}<\mu_{n}<\lambda_{n+1}
$$

After applying the above condition to the numerical experiment and discarding outliers, the corrected percentage errors for three, five and ten resonators were studied again, as presented in Fig. 10. The phenomenon of sharp increase and unstable fluctuation disappeared after correction. Without these outliers, the percentage error could be easily controlled within $0.6 \%$, regardless of the number of resonators and noise level. This demonstrates the feasibility of the proposed inverse eigenvalue analysis technique for large array applications.

\section{B. Effect of Coupling}

Parasitic coupling is a weak coupling effect widely seen in fabricated MEMS devices. This is often undesired as it can introduce crosstalk between neighbouring array elements and

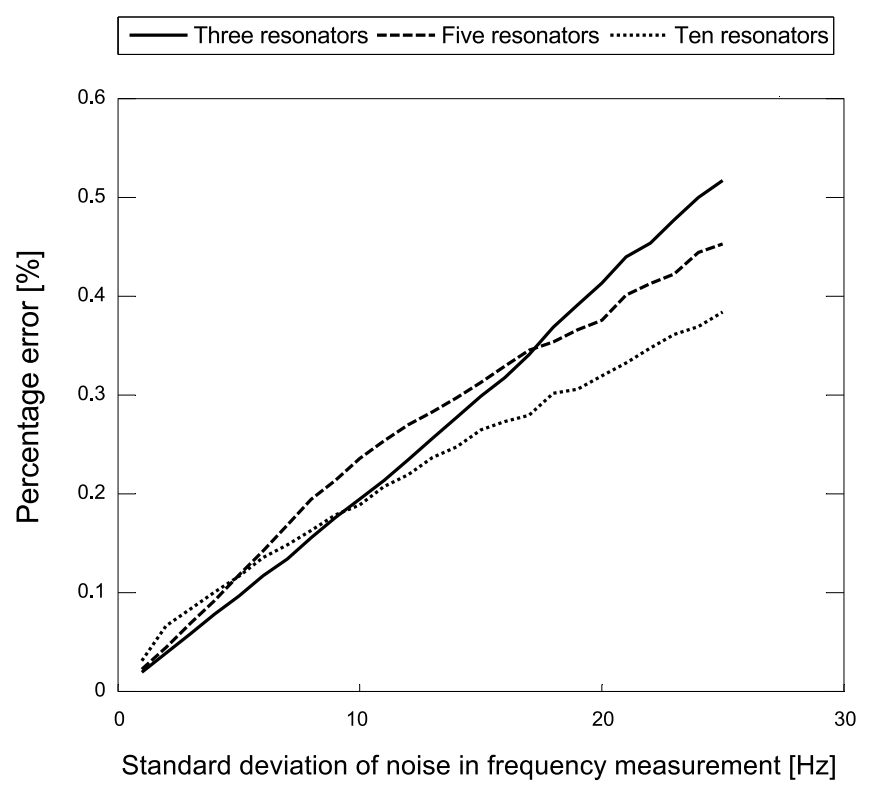

Fig. 10. Percentage error of system matrix with different noise levels for three, five and ten resonators (after correction).

degrade sensor performance. For these applications, sensors would typically be made far apart, hence wasting precious nanofabrication area. The technique proposed in this paper, however, is immune to this. Coupling is needed and welcomed to strengthen the collective behaviour of the system. Hence, the proposed technique is immune to parasitic coupling.

Coupling strength is another concern for MEMS designers [4]. Weak coupling strength is typically used to study the collective behaviour of coupled system. However, the effect of different coupling strengths has not yet been fully analysed. It is very important to maintain the precision of inverse eigenvalue analysis, therefore further investigation into the optimal coupling strength values are desired to improve the design of coupled MEMS devices. An array of three and five coupled resonators were simulated to study the effect of coupling strength, as shown in Fig. 11. The superimposed frequency noise had a standard deviation of $1 \mathrm{~Hz}$ and $2 \mathrm{~Hz}$. As the number of resonators increases, the error in extracted system elements increases. However, this can be reduced by increasing the coupling strength. Similarly, the uncertainty in results due to noise can also be reduced by increasing the coupling strength. For large array of coupled resonators, increasing the coupling strength separates the eigenvalues further apart from each other, therefore the interweaving condition in equation 17 can be more easily satisfied. This shows that coupling strength design is another way of limiting the effect of noise, as long as the collective behaviour can be correctly recorded.

\section{Damping Effect}

Experiments in section III were undertaken in low pressure, which enabled low damping, or high quality factor. However, for practical sensing applications, the air/liquid media may lower the quality factor and hence the damping in equation 1 needs to be considered. Furthermore, damping also broadens 


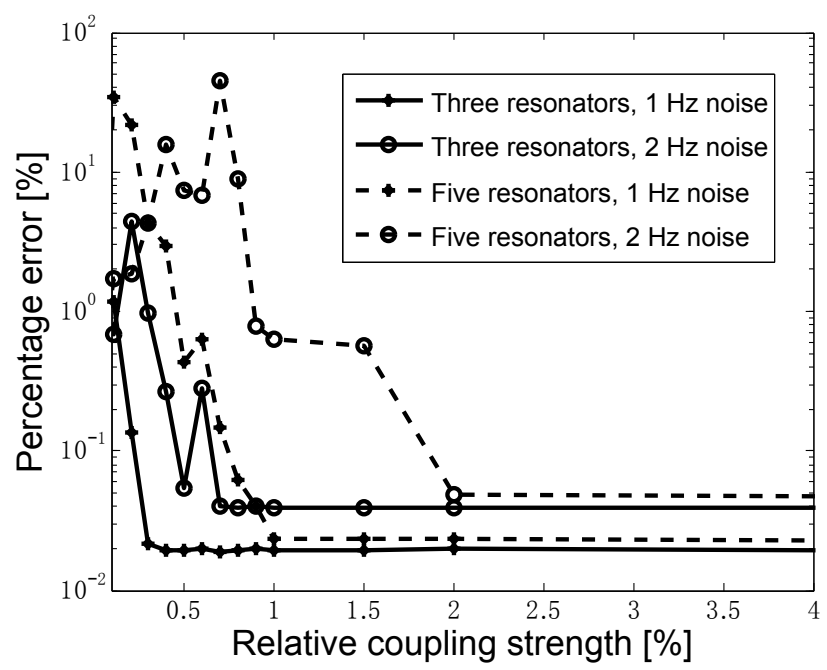

Fig. 11. Percentage error of system matrix under different coupling strengths for three and five resonators with $1 \mathrm{~Hz}$ and $2 \mathrm{~Hz}$ noise.

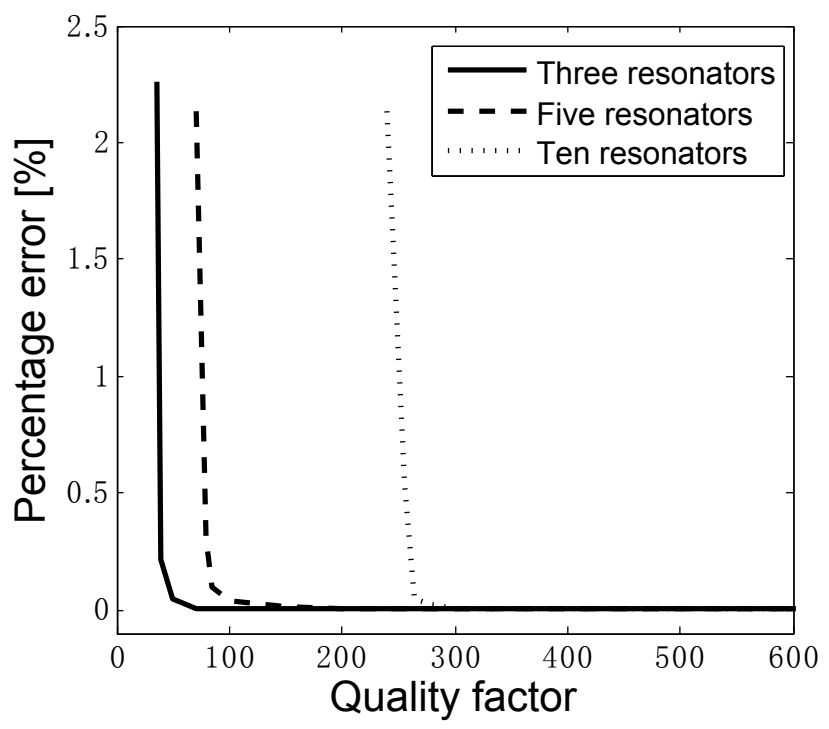

Fig. 12. Percentage error of system matrix under different quality factors for arrays with different number of resonators, each with a natural resonance frequency of $15 \mathrm{KHz}$ and a relative coupling strength of $5 \%$.

the resonance peaks of the system and could lead to overlapping peaks, which makes it difficult to identify the resonance frequencies.

Damping is known to introduce small shifts in the resonance frequencies of the system and hence the measured resonance frequencies no longer represent the eigenvalues of $\mathbf{S}_{\mathbf{n}}$. If these resonance frequencies are still used as eigenfrequencies to perform inverse eigenvalue analysis, a different system matrix $\mathbf{S}_{\mathbf{n}}^{*}$ is obtained, hence introducing error to the system. The damping error can be represented by the root mean square deviation of the relative error between the diagonal elements of $\mathbf{S}_{\mathbf{n}}^{*}$ and $\mathbf{S}_{\mathbf{n}}$ :

$$
D_{r}=\frac{\sqrt{\sum_{i=1}^{n}\left(\left|\frac{S_{n}^{*}(i, i)-S_{n}(i, i)}{S_{n}(i, i)}\right| \times 100 \%\right)^{2}}}{n}
$$

An array of three, five and ten resonators were simulated

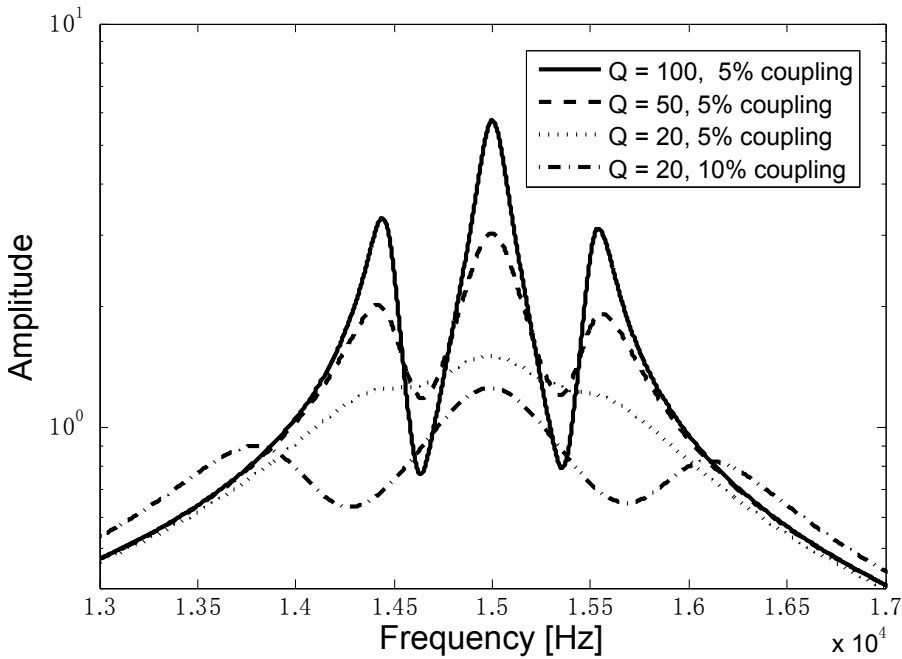

Fig. 13. Amplitude response of an array of three coupled resonators under different quality factors and coupling strengths.

respectively under different quality factors to characterize the errors introduced by damping. As shown in Fig. 12, to keep the errors comparable to the numerical error, a minimum $Q$ of around 100, 200 and 300 is required for three, five and ten resonators respectively. Hence, a system with more elements requires lower damping. If $Q$ falls below the minimum value required, the percentage error sharply increases. As quality factor further drops, damping effect makes it very difficult to record the resonance frequencies due to flattening or overlapping peaks. The experiments in this paper coupled an electrical resonator with $Q>200$ to two mechanical resonators with $Q>1000$ and hence allowed the technique to work well with very low damping error.

Fig. 13 shows the effect of damping in the frequency response of three-resonator system. As $Q$ drops from 100 to 50 , the resonance frequencies shift, thereby introducing small errors in the inverse eigenvalue analysis. When $Q$ drops further to 20 , the three peaks broaden widely and start overlapping, making the resonance frequencies unrecognisable. One solution to overcome this overlapping phenomenon is to increase the coupling strength. Increasing the relative coupling strength from $5 \%$ to $10 \%$ avoids overlapping, therefore enabling more accurate peak detection.

Hence, damping causes two major errors: one is the shift between the measured resonance frequency and actual eigenfrequency, another is the peak broadening effect which makes it difficult to accurately track the centre frequency. Under high damping conditions, the resonance peaks become overlapping and unidentifiable. However, this can be improved by increasing the coupling strength.

\section{CONClusion}

Inverse eigenvalue analysis has been used to characterize large array of coupled MEMS devices. For an array of $n$ coupled resonators, the coupling effect makes it possible to monitor $n$ resonance frequencies by driving and measuring from only one member. These $n$ resonance frequencies correspond to $n$ eigenvalues of the system matrix of the coupled 
resonators. To inversely reconstruct the system matrix, another set of eigenvalues needs to be measured by exerting change to the system. A new technique involving the coupling of an additional electrical resonator has been proposed to facilitate such change. Rather than perturbing the spring constant of the MEMS array, a second set of eigenvalues can be measured by coupling an external electrical resonator.

The new technique was experimentally verified with two coupled MEMS resonators. First, two sets of resonance frequencies were recorded before and after disconnecting the additional electrical resonator to reconstruct the system matrix. Second, after disconnecting the electrical resonator, one MEMS resonator was perturbed by applying an electrostatic force to change its effective spring constant. Through this change our previous method was used to derive the system matrix of these two MEMS devices. For further validation, perturbation analysis was once again performed towards the hybrid electrical-mechanical array. This was achieved by simply tweaking the centre frequency of the electrical circuit. The above three steps produce three system matrices of the same system, with good consistency between any two of these.

Effect of noise has been studied numerically. Monte Carlo procedure simulated different Gaussian noise levels from the frequency measurement. As the number of resonators or noise level increase, the derived system matrix becomes noisy and inaccurate. The major error arises from the algorithm failure when the frequency noise exceeds the frequency shift. This failure can be corrected by eliminating outliers which did not agree with the conditions. With the condition satisfied, the proposed technique demonstrates excellent accuracy and flexibility. Damping is another source of error as it adds additional shift to resonance frequencies and broadens the peaks.

Coupling an additional electrical resonator to MEMS resonators array is practical to implement and avoids nonlinear change or even damage to the mechanical system. Inverse eigenvalue analysis has advantages of reduced pads and connections, wider bandwidth and lower cost. It can be applied to prevent the change in manufacturing processes, test MEMS devices as well as actuating large array of sensors. In conclusion, this paper demonstrates the feasibility of the coupling between electrical and MEMS resonators. Large array of single input/output multiplexed sensors will be fabricated in the future to further validate this technique. In addition, further analysis will be carried out to fully understand the effect of damping and coupling to design optimised systems for sensing.

\section{REFERENCES}

[1] H. G. Craighead, "Nanoelectromechanical systems," Science, vol. 290, no. 5496, pp. 1532-1535, 2000.

[2] R. Datar, S. Kim, S. Jeon, P. Hesketh, S. Manalis, A. Boisen, and T. Thundat, "Cantilever sensors: nanomechanical tools for diagnostics," MRS Bull., vol. 34, no. 06, pp. 449-454, 2009.

[3] J. Wu, G. K. Fedder, and L. R. Carley, "A low-noise low-offset capacitive sensing amplifier for a $50-\mu \mathrm{g} / \mathrm{hz}$ monolithic cmos mems accelerometer," IEEE J. Solid-State Circuits, vol. 39, no. 5, pp. 722-730, May 2004.

[4] H. Zhang, B. Li, W. Yuan, M. Kraft, and H. Chang, "An acceleration sensing method based on the mode localization of weakly coupled resonators," J. Microelectromech. Syst., vol. 25, no. 2, pp. 286-296, April 2016.
[5] S. E. Alper and T. Akin, "A single-crystal silicon symmetrical and decoupled mems gyroscope on an insulating substrate," J. Microelectromech. Syst., vol. 14, no. 4, pp. 707-717, Aug 2005.

[6] C. Acar and A. M. Shkel, "Inherently robust micromachined gyroscopes with 2-dof sense-mode oscillator," J. Microelectromech. Syst., vol. 15, no. 2, pp. 380-387, April 2006.

[7] D. Weinstein, S. Bhave, M. Tada, S. Mitarai, S. Morita et al., "Mechanical coupling of $2 \mathrm{~d}$ resonator arrays for mems filter applications," in Proc. IEEE IFCS. IEEE, 2007, pp. 1362-1365.

[8] M. Zalalutdinov, J. Cross, J. Baldwin, B. Ilic, W. Zhou, B. Houston, and J. M. Parpia, "Cmos-integrated rf mems resonators," J. Microelectromech. Syst., vol. 19, no. 4, pp. 807-815, Aug 2010.

[9] D. M. Tanner, A. C. Owen, Jr., and F. Rodriguez, "Resonant frequency method for monitoring MEMS fabrication," in Proc. SPIE, vol. 4980, Jan. 2003, pp. 220-228.

[10] B. Choubey, S. Collins, and M. Ward, "On characterizing microelectromechanical processes using coupled resonators," J. Microelectromech. Syst., vol. 21, no. 4, pp. 791-800, Aug 2012.

[11] E. Boyd and D. Uttamchandani, "Measurement of the anisotropy of young's modulus in single-crystal silicon," J. Microelectromech. Syst., vol. 21, no. 1, pp. 243-249, Feb 2012.

[12] B. Choubey, E. Boyd, I. Armstrong, and D. Uttamchandani, "Determination of the anisotropy of young's modulus using a coupled microcantilever array," J. Microelectromech. Syst., vol. 21, no. 5, pp. 1252-1260, Oct 2012.

[13] A. Hajjam, J. Wilson, and S. Pourkamali, "Individual air-borne particle mass measurement using high-frequency micromechanical resonators," IEEE Sensors J., vol. 11, no. 11, pp. 2883-2890, Nov 2011.

[14] K. Park, N. Kim, D. Morisette, N. Aluru, and R. Bashir, "Resonant mems mass sensors for measurement of microdroplet evaporation," $J$. Microelectromech. Syst., vol. 21, no. 3, pp. 702-711, June 2012.

[15] M. K. Ghatkesar, V. Barwich, T. Braun, J.-P. Ramseyer, C. Gerber, M. Hegner, H. P. Lang, U. Drechsler, and M. Despont, "Higher modes of vibration increase mass sensitivity in nanomechanical microcantilevers," Nanotechnology, vol. 18, no. 44, p. 445502, 2007.

[16] J. Chaste, A. Eichler, J. Moser, G. Ceballos, R. Rurali, and A. Bachtold, "A nanomechanical mass sensor with yoctogram resolution," Nat. Nanotechnol., vol. 7, no. 5, pp. 301-304, 2012.

[17] M. S. Hanay, S. I. Kelber, C. D. O'Connell, P. Mulvaney, J. E. Sader, and M. L. Roukes, "Inertial imaging with nanomechanical systems," Nat. Nanotechnol., 2015.

[18] S. Guillon, S. Salomon, F. Seichepine, D. Dezest, F. Mathieu, A. Bouchier, L. Mazenq, C. Thibault, C. Vieu, T. Leïchlé et al., "Biological functionalization of massively parallel arrays of nanocantilevers using microcontact printing," Sens. Actuators B, Chem., vol. 161, no. 1, pp. 1135-1138, 2012.

[19] I. Bargatin, E. Myers, J. Aldridge, C. Marcoux, P. Brianceau, L. Duraffourg, E. Colinet, S. Hentz, P. Andreucci, and M. Roukes, "Largescale integration of nanoelectromechanical systems for gas sensing applications," Nano Lett., vol. 12, no. 3, pp. 1269-1274, 2012.

[20] S. Somnath, H. J. Kim, H. Hu, and W. P. King, "Parallel nanoimaging and nanolithography using a heated microcantilever array," Nanotechnology, vol. 25, no. 1, p. 014001, 2014.

[21] A. Mohammadi, S. O. R. Moheimani, and M. R. Yuce, "Parallel averaging for thermal noise mitigation in mems electrothermal displacement sensors," J. Microelectromech. Syst., vol. 24, no. 1, pp. 4-6, Feb 2015.

[22] M. Kumar and H. Bhaskaran, "Ultrasensitive room-temperature piezoresistive transduction in graphene-based nanoelectromechanical systems," Nano Lett., vol. 15, no. 4, pp. 2562-2567, 2015

[23] M. Napoli, W. Zhang, K. Turner, and B. Bamieh, "Characterization of electrostatically coupled microcantilevers," J. Microelectromech. Syst., vol. 14, no. 2, pp. 295-304, April 2005.

[24] B. E. DeMartini, J. F. Rhoads, S. W. Shaw, and K. L. Turner, "A single input-single output mass sensor based on a coupled array of microresonators," Sens. Actuators A: Phys., vol. 137, no. 1, pp. 147$156,2007$.

[25] M. Spletzer, A. Raman, H. Sumali, and J. P. Sullivan, "Highly sensitive mass detection and identification using vibration localization in coupled microcantilever arrays," Appl. Phys. Lett., vol. 92, no. 11, p. 114102, 2008.

[26] K. R. Qalandar, B. S. Strachan, B. Gibson, M. Sharma, A. Ma, S. W. Shaw, and K. L. Turner, "Frequency division using a micromechanical resonance cascade," Appl. Phys. Lett., vol. 105, no. 24, p. 244103, 2014.

[27] A. A. Glean, J. A. Judge, J. F. Vignola, and T. J. Ryan, "Mode-shapebased mass detection scheme using mechanically diverse, indirectly coupled microresonator arrays," J. Appl. Phys., vol. 117, no. 5, p. 054505, 2015. 
[28] B. Choubey, C. Anthony, N. H. Saad, M. Ward, R. Turnbull, and S. Collins, "Characterization of coupled micro/nanoresonators using inverse eigenvalue analysis," Appl. Phys. Lett., vol. 97, no. 13, p. 133114, 2010.

[29] B. E. DeMartini, J. F. Rhoads, M. A. Zielke, K. G. Owen, S. W. Shaw, and K. L. Turner, "A single input-single output coupled microresonator array for the detection and identification of multiple analytes," Appl. Phys. Lett., vol. 93, no. 5, p. 054102, 2008.

[30] J. Xu and J. M. Tsai, "A process-induced-frequency-drift resilient $32 \mathrm{khz}$ mems resonator," J. Micromech. Microeng., vol. 22, no. 10, p. 105029, 2012.

[31] A. Erbes, P. Thiruvenkatanathan, J. Woodhouse, and A. Seshia, "Numerical study of the impact of vibration localization on the motional resistance of weakly coupled mems resonators," J. Microelectromech. Syst., vol. 24, no. 4, pp. 997-1005, Aug 2015.

[32] C. De Boor and G. H. Golub, "The numerically stable reconstruction of a jacobi matrix from spectral data," Linear Algebra Appl., vol. 21, no. 3, pp. 245-260, 1978

[33] J. Tow, "Active RC filters - a state-space realization," Proc. IEEE, vol. 56, no. 6, pp. 1137-1139, June 1968.

[34] L. Thomas, "The biquad: Part I - some practical design considerations," IEEE Trans. Circuit Theory, vol. 18, no. 3, pp. 350-357, May 1971. 